\title{
Application of Particle Swarm Optimization Algorithm in Computer Neural Network
}

\author{
Xueyan Li \\ \{lixueyuanwh@126.com\}
}

City College, Wuhan University of Science and Technology Wuhan 430083,China

\begin{abstract}
Particle swarm optimization algorithm is a new intelligent optimization algorithm, which is based on the social activities of biological groups. Because the particle swarm optimization algorithm is random and uncertain, the theoretical basis of the algorithm is still not complete, for example, there are some problems, such as the premature convergence, slow convergence speed and so on. Particle swarm optimization algorithm theory analysis and improvement has become the focus and hotspot of the algorithm. Therefore, based on the theory of the traditional PSO algorithm analysis based on, combined with algorithm for biological prototype features, improved PSO algorithm is proposed for the convergence of the algorithm. Because the traditional BP artificial neural network learning algorithm is an optimization algorithm based on gradient information, algorithm is easy to fall into local optimum defects. Therefore, in this paper, the improved particle swarm optimization algorithm is used in neural network training. This study not only can improve the theoretical basis of the PSO algorithm, but also provide a reference for the application of PSO algorithm in artificial neural network.
\end{abstract}

Keywords: Particle swarm algorithm; convergence; bacteria; BP neural network

\section{Introduction}

The optimization problem is a based in mathematics and solve various engineering problems for optimal solutions of a kind of application, the content is to discuss in a large number of projects to find out the optimal scheme, and how to find the optimal solution. Optimization problem can also be defined as follows: in order to satisfy certain constraints, to find a set of parameter values, so that the system can achieve the maximum value or minimum value. Optimization problem is a kind of frequently encountered problem in many fields, such as scientific research, engineering technology, economic management, production process and so on. Optimization problem is an old topic. For a long time, people have made unremitting efforts to explore and study the optimization problems. Early in the 17th century, Newton in the UK and Germany Leibnitz invented the calculus contains optimization problems, and the French mathematician Cauchy is for the first time using gradient descent method for solving unconstrained optimization problems appeared, and then the Lagrange multiplier method to solve optimization problems [1]. It was not until 1947 that Dantzig proposed the simplex method for solving the general linear programming problem. With the rapid development of science and technology, especially high speed digital computer, it has promoted the rapid development of the optimization method, and it has penetrated into every field. Has appeared 
in the linear programming, integer programming, non - new planning, assembly planning, and many other branches. These optimization techniques also play a more and more role in the practical application. Optimization theory and algorithm research is an important subject which has both theoretical significance and applied value. With the development of science and technology and society, numerical method is applied to solve the problem of the development of simulating biological group behavior characteristic, things and design optimization algorithm in solving optimization, the basic iterative algorithm update is relatively simple, but in general, the number of iteration is relatively large, but with the advent of the computer, for simple and repetitive operation provides guaranteed to make the algorithm get the rapid development, this kind of algorithm is generally referred to as evolutionary algorithms. These algorithms mainly include artificial neural network (ANN), simulated annealing algorithm (SA), genetic algorithm (GA), ant colony algorithm (ACO), particle swarm optimization (PSO) and so on. These algorithms have a common characteristic: it is through simulation or reveal some nature phenomena and process development and in the optimization problem areas, known as intelligent optimization algorithm (intelligent optimization algorithms) [2].

\section{The Basis of Particle Swarm Optimization}

Particle swarm optimization algorithm is an optimization algorithm proposed by American social psychologist Kennedy James and electrical engineer Eberhart Russell. They find that the cooperation and competition among individuals in a biological colony can produce cluster intelligence, which can provide effective solutions to some specific problems. Birds in the process of searching for food, the exchange and sharing of information between individuals can be carried out, each member can benefit from all other members of the discovery and flight experience. The advantage of this collaboration is decisive when the food source is unpredictable and sporadic, far greater than the disadvantage of competition for food. Biologists run computer simulations on the birds, and they allow each individual to follow a particular rule to form a complex behavior of birds. Model in the key point is to the operation of the distance between the individual, namely the individual efforts to maintain their distance between neighbors and is the best, so as to achieve the synchronization of the group behavior, for each individual must know its location and neighbor information. This is the origin of the idea of particle swarm optimization algorithm. Particle swarm optimization (PSO) algorithm has the advantages of simple structure, less parameters and fast convergence advantages. Since then it quickly by the attention of domestic and foreign scholars, the soon to be widely application in different fields, and has obtained the good effect. This chapter will introduce the concept of particle swarm optimization algorithm, the principle of the algorithm and the process of the algorithm. Focuses on the three typical particle group optimization algorithm, which with the inertia weight particle group optimization algorithms are also known as the standard particle swarm algorithm, later scholars put forward a to ensure the convergence of the particle swarm optimization algorithm, called with a constriction factor particle swarm optimization algorithm. Finally introduced five benchmark functions. Due to the algorithm performance, as at present, most scholars use different types of function test requirements of the algorithm as a function of the different types of different, this chapter selection of several typical benchmark functions, multi peak function more are with many of the local extremum. 
In this way, the requirements of the algorithm is relatively high, and at the same time, several test functions are also one of the criteria to test the performance of the algorithm.

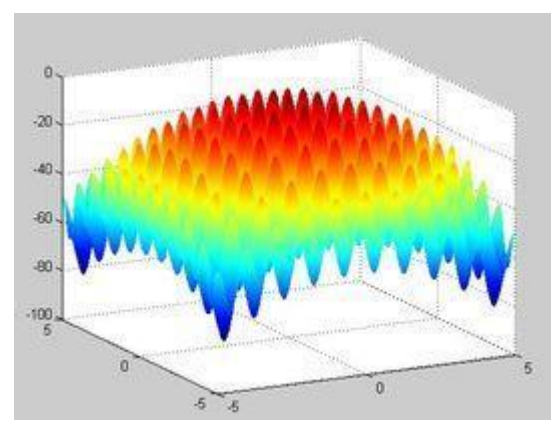

Fig. 1. Schematic diagram of particle swarm algorithm

\subsection{The Concept of Particle Swarm Optimization Algorithm}

Individual search needs to be carried out in a group, which is composed of a number of individual groups, namely, a population (or a group). At any time of the particle swarm optimization algorithm, there are many individuals, which represent the multiple solutions of the optimization problem. Population size is one of the main parameters of particle swarm optimization algorithm, and the setting of population size may affect the optimization effect of particle swarm optimization algorithm. Population size is too small, so that the population volatile diversity capacity, which affects the ability of the global search algorithm; population size is too large, the algorithm is easy to degenerate into a search. Although there are many research results on the population scale of study, and gives the recommended value, but a large number of simulation experiments results show that population size settings and to be optimized problem, particle swarm optimization of other parameters, the problem size and has has a close relationship. Particle swarm optimization (PSO) algorithm is derived from the foraging behavior of birds. Kennedy and Eberhart found birds in flight process often suddenly changed direction [3], spread, aggregation, its behavior is unpredictable, but their overall always maintain consistency, between the individual and the individual also maintained a distance to the optimum. Through the research on the behavior of similar biological groups, it is found that there is a kind of social information sharing mechanism, which provides an advantage for the evolution of the population.

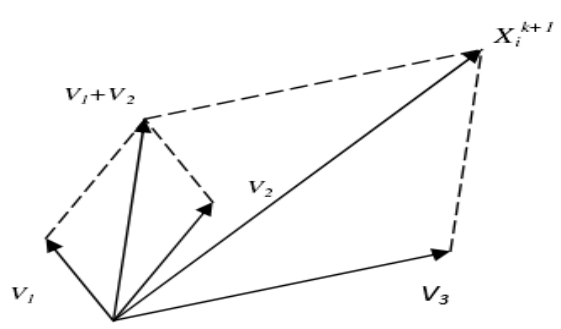


Fig. 2. Particle position update

\subsection{Reference Function}

Function optimization problem is a common example to test the algorithm. Due to the randomness of particle swarm algorithm and uncertainties particles prone to the phenomenon of premature convergence and local optimum, also in the process of the search algorithm and may appear to function optimization problems is to test the algorithm commonly used examples. Although the particle swarm optimization algorithm has many improvements and deformation, but the improved particle group optimization algorithm have features in common with, that is, by on individual optimal and global optimal updating and learning, constantly adjust the flight speed of particles to update location, complete to search the solution of the optimization problem [4].

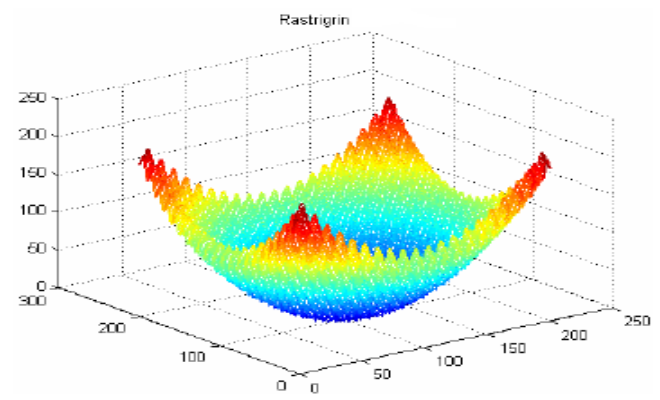

Fig. 3. Three dimensional graph of function

\section{Improvement of Particle Swarm Optimization Algorithm}

PSO algorithm's biggest shortcoming is the premature convergence problem, namely when a particle is found a current best position, other particles quickly to its close, if the location for the local advantages, particle swarm difficult again solution space to search, thus fall into local convergence. At present, measures to overcome the PSO algorithm premature convergence is 4: 1 . Adjust parameter selection strategy, equilibrium particle of global and local search ability; (2) to increase particle swarm size to extend the solution space; (3) in a limited scale keep the diversity of particle swarm, in the evolutionary process in continuous search in new spaces; (4) mixed with a variety of heuristic algorithm to enhance particle local search ability. Increase in particle swarm size although it can obtain the better solution, but a substantial increase in the computational complexity of the algorithm, moreover is difficult to fundamentally overcome the local convergence problem, after reaching a certain scale cannot to improve the search ability of the algorithm, so parameter adjustment strategy need after a large number of simulation experiments on specific issues can be determined and all the improvement strategy could not apply to all of the optimization problem. Also hybrid PSO algorithm in accordance with the particle diversity of change adjust disturbance factor in enhanced the ability of local search algorithm and particle swarm diversity at the same time, it 
also makes the algorithm in each evolution process increases the amount of computations. This will affect the PSO's rapid convergence ability. In this chapter, an improved particle swarm optimization algorithm (ICFPSO) based on enhanced particle swarm optimization (PSO) is proposed, and a hybrid optimization algorithm is proposed to enhance the local search capability [5].

The first improvement strategy is with constriction factor particle group optimization algorithm based on increased two new parameters and to determine whether the particles appear stagnation phenomenon, when there is a problem of premature convergence by re initialize the particle position strategy increase the diversity of particles, effectively improves the convergence rate of the algorithm.

The second improvement method is using bacterial evolutionary mechanisms and of particle swarm optimization algorithm is improved. The bacteria as biological prototype of individuals in the algorithm, and put forward a kind of evolutionary mechanism of bacterial particle swarm algorithm based on bacterial colony and the basic biological evolution, which individuals through constant propagation and killed to keep the population diversity, the algorithm for particle swarm optimization algorithm put forward a kind of new natural end. The simulation results show that the algorithm can significantly improve the convergence speed, and can achieve the global optimum.

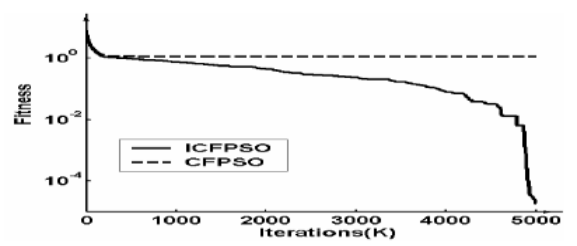

Fig. 4. Ackley function comparison curve

\section{Conclusion}

In this paper, the origin of particle swarm optimization algorithm, the convergence analysis of the algorithm, the improvement of the algorithm and its application in neural network are studied and discussed. On the basis of analyzing the effect of algorithm parameters on the convergence, convergence speed and accuracy; on particle swarm optimization algorithm of behavior analysis, and gives the algorithm convergence region parameters. For the following study provide criterion for the selection of the parameters; finally, it is proved that the algorithm convergence location of existence and uniqueness.

\section{Acknowledgements}

The Education Department Foundation of Hubei Province of China under Grant No. B2015362. 


\section{References}

[1] Zhang Hongli, Li Ruiguo, Wang Ya. Chaotic time series prediction based on Hermite neural network. Computer application and software. No. 04. (2016)

[2] Liu Hongbin, Xiong Wentao, Li Huawen. Optimization of sheet metal forming based on GS theory and neural network genetic algorithm function optimization. Mechanical design and research. No. 02. (2016)

[3] Yuan Zeyi, Zhu Jiaming, Li Zhenguo, Li Dongling. Quantitative analysis of the sewage treatment based on neural network. Journal of Jiamusi University (NATURAL SCIENCE EDITION). No. 02. (2016)

[4] Gong Dunwei, Yao Xiangjuan, Jeky. Integrated neural network into the path covering test data evolution generation. Software Journal. No. 04. (2016)

[5] Zhang Jianing. Study on the urban traffic flow prediction model based on neural network and similar days. Industrial control computer. No. 04. (2016) 